\title{
INTEGRABLE MEAN PERIODIC FUNCTIONS ON LOCALLY COMPACT ABELIAN GROUPS
}

\author{
INDER K. RANA AND K. GOWRI NAVADA
}

(Communicated by J. Marshall Ash)

\begin{abstract}
Let $G$ be a locally compact abelian group with a Haar measure $\lambda_{G}$. A function $f$ on $G$ is said to be mean-periodic if there exists a nonzero finite regular measure $\mu$ of compact support on $G$ such that $f * \mu=0$. It is known that there exist no nontrivial integrable mean periodic functions on $\mathbf{R}^{n}$. We show that there exist nontrivial integrable mean periodic functions on $G$ provided $G$ has nontrivial proper compact subgroups. Let $f \in L_{1}(G)$ be mean periodic with respect to a nonzero finite measure $\mu$ of compact support. If $\mu(G) \neq 0$ and $\lambda_{G}(\operatorname{supp}(\mu))>0$, then there exists a compact subgroup $K$ of $G$ such that $f * \lambda_{K}=0$, i.e., $f$ is mean periodic with respect to $\lambda_{K}$, where $\lambda_{K}$ denotes the normalized Haar measure of $K$. When $G$ is compact, abelian and meterizable, we show that there exists continuous (hence integrable and almost periodic) functions on $G$ that are not mean periodic.
\end{abstract}

\section{INTRODUCTION}

The theory of mean periodic functions on the real line $\mathbf{R}$ was initiated by Delsarte [2, 3] and were analyzed in detail by Schwartz [11], Kahane [7], and Malgrange [8] on $\mathbf{R}^{n}$ (see also Berenstein and Taylor [1] for mean periodic functions on $\mathbf{R}^{n}$ and other references). Ehrenpreis and Mautner [5] started the study of mean periodic functions on the group $\operatorname{SL}(2, \mathbf{R})$. It is well known that there does not exist nontrivial integrable mean periodic functions on $\mathbf{R}^{n}$. However, Sitaram [12] has given an example of a nontrivial integrable mean periodic function on $\operatorname{SL}(2, \mathbf{R})$. The aim of this note is to analyze mean periodic integrable functions on locally compact abelian groups.

Let $G$ be a locally compact abelian group with a Haar measure $\lambda_{G}$. We shall denote by $M(G)$ the set of all finite regular measures on $G$, and $M_{c}(G)$ will denote the set of all $\mu \in M(G), \mu$ with compact support. $L_{1}(G)$ denotes the space of all integrable functions on $G$.

1.1. Definition. We call $f \in L_{1}(G)$ mean periodic if there exists $\mu \in M_{c}(G)$, $\mu \neq 0$, such that $f * \mu=0$; here * denotes the convolution operation. We shall also say $f$ is mean periodic with respect to $\mu$.

Received by the editors September 24, 1990 and, in revised form, June 6, 1991.

1991 Mathematics Subject Classification. Primary 43A70; Secondary 42A75.

Key words and phrases. Locally compact abelian groups, mean periodic functions on groups, almost periodic functions, character group, annihilator, Fourier transform. 
1.2. Example. Let $K$ be any compact abelian group, and let $\widehat{K}$ denote the dual group of $K$. Let $\gamma \in \widehat{K}$ be any nontrivial character. Then for $x \in K$,

$$
\left(\gamma * \lambda_{K}\right)(x)=\int\langle x-y, \gamma\rangle d \lambda_{K}(y)=\langle x, \gamma\rangle \int\langle\overline{y, \gamma}\rangle d \lambda_{K}(y) .
$$

Also $\int_{K}\langle x-y, \gamma\rangle d \lambda_{K}(x)=\int_{K}\langle\overline{y, \gamma}\rangle d \lambda_{K}(y)$. Since $\gamma$ is nontrivial, we have $\int_{K}\langle\overline{y, \gamma}\rangle d \lambda_{K}(y)=0$, i.e., $\gamma * \lambda_{K}=0$.

Thus every nontrivial $\gamma \in \widehat{K}$ is mean periodic. Also if $f$ is any constant function on $K$ and $K$ has at least two distinct points $k_{1}, k_{2} \in K$, then $f *$ $\left(\delta_{k_{1}}-\delta_{k_{2}}\right)=0$, where $\delta_{k}$ denotes the unit mass at $k$.

1.3. Example. Let $\mathbf{T}$ denote the circle group and $f(x)=x, x \in \mathbf{T}$. We have already seen in Example 1.2 that $f$ is mean periodic with respect to $\lambda_{\mathbf{T}}$, the normalized Haar measure on $\mathbf{T}$. Let $\nu$ denote the measure on $\mathbf{T}$ given by $d \nu(x)=1 / x d \lambda_{\mathbf{T}}(x)$. Then it is easy to see that $f * \nu=0$, i.e., $f$ is mean periodic with respect to $\nu$.

1.4. Example. Let $G$ be a locally compact abelian group such that $G$ has a nontrivial proper compact subgroup $K$. Let $\Lambda=\{\gamma \in \widehat{G} \mid\langle x, \gamma\rangle=0$ for all $x \in K\}$ denote the annihilator of $K$. Then $\Lambda$ is a proper closed subgroup of $\widehat{G}$. Let $\gamma_{0} \in \widehat{G} \backslash \Lambda$. Choose a neighbourhood $U$ of $\gamma_{0}$ and $V$ of $\Lambda$ such that $U \cap V=\varnothing$. Then $\gamma_{0} \in U \subseteq V^{c}$. Now choose $f \in L_{1}(G)$ such that $\hat{f}\left(\gamma_{0}\right)=1$ and $\hat{f}(\gamma)=0$ for every $\gamma \in U^{c}$ (see [11, Theorem 2.6.2]). Thus $\hat{f}(\gamma)=0$ for every $\gamma \in \Lambda$, and hence $f * \lambda_{K}=0$ (see [10, Theorem 2.7.4]). Thus, we have shown that there exists nontrivial $f \in L_{1}(G)$ that are mean periodic provided $G$ has nontrivial proper compact subgroups.

We next analyze integrable mean periodic functions on compact abelian groups.

\section{INTEGRABLE MEAN PERIODIC FUNCTIONS ON COMPACT ABELIAN GROUPS}

Let $K$ be a nontrivial compact abelian group. In view of Example 1.2, one may ask: is every $f \in L_{1}(K)$ mean periodic? We shall see that this is not so in general. However, every $f \in L_{1}(K)$ can be written as a sum of two integrable mean periodic functions on $K$.

2.1. Proposition. Let $K$ be a compact abelian group and $f \in L_{1}(K)$. Then $f=g+h$, where $g, h$ are mean periodic functions in $L_{1}(K)$.

Proof. Let $\lambda_{K}$ denote the normalized Haar measure of $K$. Let $h=f * \lambda_{K}$ and $g=f-h$. Then clearly $g * \lambda_{K}=0$ and $h(x)=\int_{K} f d \lambda_{K}$ for every $x \in K$. Thus $g, h$ are mean periodic functions in $L_{1}(K)$ and $f=g+h$.

2.2. Remark. The above proposition says that $L_{1}(K)=S+C$, where $S, C \subseteq$ $L_{1}(K)$ and every $f \in S \cup C$ is a mean periodic function. In fact, $C$ is the space of all constant functions on $K$. One can say something more about $S$. We claim that $S=\left\{g \in L_{1}(K) \mid g * \lambda_{K}=0\right\}$ is a maximal ideal of $L_{1}(K)$. To see this we first note that $S$ is a closed subalgebra of $L_{1}(K)$. Next, if $\gamma_{0} \in \widehat{K}$ and $\gamma_{0} \not \equiv 1$, we choose a neighbourhood $W$ of $\gamma_{0}$ such that $1 \notin W$. Now we choose $g \in L_{1}(K)$ such that $\hat{g}\left(\gamma_{0}\right)=1$ and $\hat{g}(\gamma)=0$ for every $\gamma \in W^{c}$ (see [10, Theorem 2.6.2]). Thus, $\hat{g}\left(\gamma_{0}\right)=1$ and $\hat{g}(1)=0$. But then 
$\left(g * \lambda_{K}\right)(x)=\int_{K} g(x-y) d \lambda_{K}(y)=\int_{K} g(y) d \lambda_{K}(y)=\hat{g}(1)=0$. Thus, for $g \in S, \hat{g}$ separates points of $\widehat{K}$. Hence by a corollary [10, p. 232] either $S=L_{1}(K)$ or $S$ is a maximal ideal of $L_{1}(K)$. Since $S \neq L_{1}(K), S$ is a maximal ideal of $L_{1}(K)$.

We show next that not every $f \in L_{1}(K)$ is mean periodic.

2.3. Proposition. Let $K$ be a compact metrizable abelian group and let $\widehat{K}$ be its dual group. Let $f=\sum_{\gamma \in \widehat{K}} a_{\gamma} \gamma$, where $a_{\gamma} \in \mathbf{C}$ and the series converges uniformly on $K$. Then there exists a nonzero finite measure $\mu$ on $K$ such that $f * \mu=0$ iff $a_{\gamma}=0$ for at least one $\gamma \in \widehat{K}$.

Proof. Clearly $a_{\gamma}=\hat{f}(\gamma)$ for every $\gamma \in \widehat{K}$. Further $f * \mu=\sum_{\gamma \in \widehat{K}} a_{\gamma} \hat{\mu}(\gamma) \gamma$. Now if $f * \mu=0$, then for every $\gamma \in \widehat{K}, 0=(f \hat{*} \mu)(\gamma)=\hat{f}(\gamma) \hat{\mu}(\gamma)=a_{\gamma} \hat{\mu}(\gamma)$. Since $\mu \neq 0$, there exists at least one $\gamma_{0} \in \widehat{K}$ such that $\hat{\mu}\left(\gamma_{0}\right) \neq 0$. Thus, $a_{\gamma_{0}}=0$. Conversely, if $a_{\gamma_{0}}=0$ for some $\gamma_{0} \in \widehat{K}$, then $f * \mu=0$ for $d \mu(x)=$ $\gamma_{0}(x) d \lambda_{K}(x)$.

2.4. Corollary. Let $K$ be a compact metrizable abelian group and let $\widehat{K}$ be its dual group. Let $f=\sum_{\gamma \in \widehat{K}} a_{\gamma} \gamma$ where $0 \neq a_{\gamma} \in \mathbf{C}$ for every $\gamma \in \widehat{K}$ and the series converges uniformly. Then $f$ is a continuous hence integrable (and almost periodic) function on $K$ and $f$ is not mean periodic.

2.5. Remark. Corollary 2.4 extends a remark of Kahane [7] that on $\mathbf{R}^{n}$ there exist almost periodic functions that are not mean periodic.

\section{INTEGRABLE MEAN PERIODIC FUNCTIONS ON LOCALLY COMPACT ABELIAN GROUPS}

Let $G$ be a locally compact abelian group. As seen in Example 1.4, if $G$ has a nontrivial compact subgroup $K$, then there exists $0 \neq f \in L_{1}(G)$ that is mean periodic. To be more precise, $f * \lambda_{K}=0$, where $\lambda_{K}$ denotes the normalized Haar measure of $K$. We ask the question: if $f \in L_{1}(G)$ and is mean periodic, under what conditions can one say $f * \lambda_{K}=0$ for some compact subgroup $K$ of $G$ ? The answer is given by the following

3.1. Theorem. Let $G$ be a locally compact abelian group and $f \in L_{1}(G)$ be mean periodic with respect to $0 \neq \mu \in M_{c}(G)$. If $\mu(G) \neq 0$ and $\lambda_{G}(\operatorname{supp}(\mu))>$ 0 , then there exists a compact subgroup $K$ of $G$ such that $f * \lambda_{K}=0$.

To prove the theorem, we need some preliminary results. Let $G$ be a locally compact abelian group and $K$ a compact subgroup of $g$. Let $\lambda_{G}, \lambda_{K}$, and $\lambda_{G / K}$ denote the Haar measures of $G, K$, and $G / K$, respectively, such that $\lambda_{K}(K)=1$ and such that for every $f \in L_{1}(G)$

$$
\int_{G} f d \lambda_{G}=\int_{G / K}\left(\int_{K} f(x+y) d \lambda_{K}(y)\right) d \lambda_{G / K} .
$$

Let $M(G)$ and $M(G / K)$ denote the space of all bounded regular measures on $G$ and $G / K$, respectively. Following [10, §2.7], we define

$$
\begin{gathered}
T: M(G) \rightarrow M(G / K), \\
(T \mu)(f)=\int_{G}(f \circ \Pi)(x) d \mu(x), \quad f \in C_{0}(G / K), \mu \in M(G),
\end{gathered}
$$


where $\Pi: G \rightarrow G / K$ is the natural homomorphism and $C_{0}(G / K)$ denotes the space of all continuous functions on $G / K$ vanishing at $\infty$. Given $\mu$, $T \mu \in M(G / K)$ is well defined and

$$
\int_{G}(f \circ \Pi)(x) d \mu(x)=\int_{G / K} f d(t \mu)
$$

for every bounded Borel function $f$ on $G / K$. The following properties of $T$ are easy to check.

3.2. Proposition. (i) If $\mu \in M(G)$ is such that either $\mu(G) \neq 0$ or $\mu \neq 0$ and $\mu^{\prime}$ is $K$-invariant, then $T(\mu) \neq 0$.

(ii) If $\mu \in M(G)$ has compact support, then so does $T \mu$.

(iii) For $f \in L_{1}(G), T f \in L_{1}(G / K)$ and

$$
(T f)(\Pi(x))=\int_{K} f(x+k) d \lambda_{K}(k), \quad x \in G .
$$

(iv) For $f \in L_{1}(G)$ and $\mu \in M_{c}(G), T(f * \mu)=(T f) *(\mu)$.

(v) If $f \in L_{1}(G)$ is $K$-invariant and $(T f)(\Pi(x))=0$ for every $x \in G$, then $f(x)=0$ for every $x \in G$.

Our next lemma is motivated by the lemma of $[9$, p. 311]

3.3. Lemma. Let $f \in L_{1}(G)$ be a continuous function and $\mu \in M_{c}(G)$ be such that $\mu(G) \neq 0, \lambda_{G}(\operatorname{supp}(\mu))>0$, and $f * \mu=0$. Then there exists a compact subgroup $K$ of $G$ such that $f * \lambda_{K}=0$.

Proof. Let $E=\operatorname{supp}(\mu)$. Then $E$ is a compact subset of $G$ and $\lambda_{G}(E)>0$. Let $G_{0}$ be the subgroup of $G$ generated by $E$. Then $G_{0}$ is a locally compact compactly generated abelian group and, by the structure theorem (Hewitt and Ross [6]), $G_{0}$ is topologically isomorphic to $\mathbf{R}^{n} \times \mathbf{Z}^{k} \times K$, where $\mathbf{Z}$ denotes the integer group, $n, k$, are nonnegative integers, and $K$ is a compact abelian group. We assume without loss of generality that $G_{0}=\mathbf{R}^{n} \times \mathbf{Z}^{k} \times K$. To prove the lemma, we first assume that $f$ is $K$-invariant, i.e., $f(x+k)=f(x)$ for all $x \in G, k \in K$. We shall show $f * \lambda_{K}=f \equiv 0$. Choose $x_{0} \in G$ arbitrarily and fix it. Let $f_{x_{0}}(x)=f\left(x+x_{0}\right), x \in G$. Then $f_{x_{0}}$ is a $K$-invariant continuous integrable function on $G$ and $f_{x_{0}} * \mu=0$. Since $\mu$ is concentrated on $G_{0}$, this gives $f_{x_{0}} * \mu=0$ on $G_{0}$. Using Proposition 3.2,

$$
T\left(f_{x_{0}} * \mu\right)=T\left(f_{x_{0}}\right) * T(\mu)=0 \text { on } \mathbf{R}^{n} \times \mathbf{Z}^{k} .
$$

Taking the Fourier transform, we have

$$
\left(\widehat{T f}_{x_{0}}\right)(\gamma) \cdot(\widehat{T(\mu)})(\gamma)=0 \text { for every } \gamma \in \mathbf{R}^{n} \times \mathbf{T}^{k} \text {. }
$$

Since $\mu \in M_{c}(G)$ and $\mu\left(G_{0}\right) \neq 0$, again by Proposition 3.2, $0 \neq T \mu \in$ $M_{c}\left(G_{0} / K\right)=M_{c}\left(\mathbf{R}^{n} \times \mathbf{Z}^{k}\right)$. Thus $(\widehat{T \mu})(\gamma) \neq 0$ for almost all $\gamma \in \mathbf{R}^{n} \times \mathbf{T}^{k}$. Hence $(* *)$ gives $\left(\widehat{T f}_{x_{0}}\right)(\gamma)=0$ for almost all $\gamma \in \mathbf{R}^{n} \times \mathbf{T}^{k}$. Thus, $\left(T f_{x_{0}}\right)(\bar{x})=$ 0 for almost all $\bar{x} \in \mathbf{R}^{n} \times \mathbf{Z}^{k}$. Since $f_{x_{0}}$ is continuous, this implies $T f_{x_{0}}(\bar{x})=0$ for all $\bar{x} \in G_{0} / K=\mathbf{R}^{n} \times \mathbf{Z}^{k}$. Again, since $f_{x_{0}}$ is $K$-invariant, by Proposition 3.2 , this implies that $f_{x_{0}}(x) \equiv 0$. To prove the proposition in the general case, we consider the function $f * \lambda_{K}$ and apply the above case to conclude $f * \lambda_{K} \equiv 0$.

Proof of Theorem 3.1. Let $f \in L_{1}(G)$ and $\mu \in M_{c}(G)$ be such that $\mu(G) \neq 0$ and $f * \mu=0$. Let $\phi \in C_{c}(G)$, the space of continuous functions on $G$ with 
compact support. Then $(f * \mu) * \phi=(f * \phi) * \mu=0$ on $G$. Since $f * \phi \in L_{1}(G)$ is a continuous function, by Lemma 3.3, $(f * \phi) * \lambda_{K}=0$ for all $\phi \in C_{c}(G)$. Thus, $(f * \lambda) * \phi=0$ for all $\phi \in C(G)$ and hence $f * \lambda=0$. This proves the theorem.

3.4. Remark. The condition that $\mu(G) \neq 0$ in Theorem 3.1 can be replaced by a weaker condition: $\mu * \lambda_{K} \neq 0$, where $K$ is the maximal compact subgroup of $G_{0}$, the subgroup of $G$ generated by $\operatorname{supp}(\mu)$. To see this we note that in the proof of Lemma 3.3, the equation $(*)$ can be replaced by

$$
T\left(f_{x_{0}} * \mu * \lambda_{K}\right)=T\left(f_{x_{0}}\right) * T \tilde{\mu}=0,
$$

where $\tilde{\mu}=\mu * \lambda_{K}$. Since $\tilde{\mu} \neq 0$ and is $K$-invariant, by Proposition 3.2, $T(\tilde{\mu}) \neq 0$ has compact support. Now proceeding as in Lemma 3.3, we will get Lemma 3.1 under the condition $\mu * \lambda_{K} \neq 0$. Hence Theorem 3.1 will hold under the conditions: $\mu * \lambda_{K} \neq 0$ and $\lambda_{G}(\operatorname{supp}(\mu))>0$.

In the next theorem, we give some equivalent versions of the conclusions of Theorem 3.1.

3.5. Theorem. Let $G$ be a locally compact abelian group. Let $f \in L_{1}(G)$ be mean periodic with respect to a measure $\mu \in M_{c}(G)$, where $\mu(G) \neq 0$ and $\lambda_{G}(\operatorname{supp}(\mu))>0$. Then the following hold:

(i) $f$ is mean periodic with respect to the normalized Harr measure $\lambda_{K}$ of some compact subgroup $K$ of $G$.

(ii) There exists a compact subgroup $K$ of $G$ such that $\hat{f}(\gamma)=0$ for all $\gamma \in \operatorname{Ann}(K)$.

(iii) There exists a compact subgroup $K$ of $G$ such that

$$
f \in \operatorname{cl}\left\{g \in L_{1}(G) \mid \operatorname{Ann}(K) \subset \operatorname{Int} Z(g)\right\},
$$

where cl denotes the closure in $L_{1}$-norm, $Z(g)=\{\gamma \in \widehat{G} \mid \hat{g}(\gamma)=0\}$, and Int $Z(g)$ denotes the interior of $Z(g)$.

Proof. Under the given conditions, clearly (i) holds by Theorem 3.1. We shall show (i) $\Rightarrow$ (ii) $\Leftrightarrow$ (iii). Implication (i) $\Rightarrow$ (ii) is Theorem 2.7.4 of [10]. Suppose now (ii) holds. Since $\Lambda=\operatorname{Ann}(K)$ is a closed subgroup of $G$, by Theorem 7.5.2(d) of [10], it follows that for every $\varepsilon>0$, there exists $g \in L_{1}(G)$ such that $\hat{g}$ has compact support disjoint from $\Lambda$ and $\|f-f * g\|<\varepsilon$. Further $f * g \in I_{0}(\Lambda)=\operatorname{cl}\left\{\phi \in L_{1}(G) \mid \Lambda \subset \operatorname{Int} Z(\phi)\right\}$ by Theorem 7.5.2(a) of [10]. But then clearly $f \in I_{0}(\Lambda)$. This proves (ii) $\Rightarrow$ (iii). Also if $f \in I_{0}(\Lambda)$, clearly $\Lambda \subset Z(f)$ and thus (iii) $\Rightarrow$ (ii).

\section{ACKNOWLEDGMENT}

The authors would like to thank the referee for the arguments presented in Proposition 2.3.

\section{REFERENCES}

1. C. A. Berenstein and B. A. Taylor, Mean periodic functions, Internat. J. Math. Math. Sci. 3 (1980), 195-235.

2. J. Delsarte, Les fonctions moyenne-périodiques, J. Math. Pures Appl. 14 (1935), 403-453.

3. _ Théorie des fonctions moyenne-périodiques de deux variables, Ann. of Math. (2) 72 (1960), 121-178. 
4. L. Ehrenpreis, Mean periodic functions, Amer. J. Math. 77 (1955), 293-328.

5. L. Ehrenpreis and F. I. Mautner, Some properties of Fourier transform on semi-simple Lie groups. II, Trans. Amer. Math. Soc. 84 (1957), 1-55.

6. E. Hewitt and K. A. Ross, Abstract harmonic analysis. I, Springer-Verlag, Berlin and New York, 1963.

7. J. P. Kahane, Lectures on mean periodic functions, Tata Institute of Fundamental Research, Bombay, 1957.

8. B. Malgrange, Existence et approximation des solutions des équations aux dérivées partielles et des équations de convolution, Ann. Inst. Fourier (Grenoble) 6 (1955/56), 271-368.

9. Inder K. Rana, A uniquenes problem for probability measures on locally-compact Abelian groups, Sankhyā Ser. A 39 (1977), 309-316.

10. W. Rudin, Fourier analysis on groups, Interscience, New York, 1967.

11. L. Schwartz, Théorie générale des fonctions moyenne-périodiques, Ann. of Math. (2) 48 (1947), 55-79.

12. A. Sitaram, Some remarks on measures on non-compact semi-simple Lie groups, Pacific J. Math. 110 (1984), 429-434.

Department of Mathematics, Indian Institute of Technology, Powai, Bombay 400076, INDIA 